\title{
N-Myc downstream-regulated gene 2 suppresses the proliferation of T24 human bladder cancer cells via induction of oncosis
}

\author{
JIE HUANG, ZHOU WU, GUANGXIU WANG, YINGXIAN CAI, MINSHAN CAI and YAOZHANG LI \\ Cancer Center, Affiliated Hospital of Guangdong Medical College, Zhanjiang, Guangdong 524001, P.R. China
}

Received June 27, 2014; Accepted March 10, 2015

DOI: $10.3892 / \mathrm{mmr} .2015 .4169$

\begin{abstract}
Previous studies have reported the antitumor activity of N-Myc downstream-regulated gene 2 (NDRG2), a novel p53-inducible gene, in several types of cancer. The present study aimed to investigate the effects of NDRG2 expression on the proliferation of a human bladder cancer cell line. NDRG2 and control green fluorescent protein (GFP) recombinant adenovirus plasmids were constructed and transfected into a bladder cancer cell line with mutant p53 (T24 cells). NDRG2 expression was analyzed using western blot analysis and immunofluorescence assay (IFA); in addition, the subcellular localization of NDRG2 was detected using a confocal microscope. The proliferation rate of cells was measured using colony formation and MTT assays. Furthermore, the cell cycle of transfected T24 cells was detected by flow cytometry. The results indicated that T24 cells expressed low levels of NDRG2 prior to infection with GFP-NDRG2 recombinant adenovirus; by contrast, following infection, NDRG2 was primarily overexpressed in mitochondria. The proliferation rate of T24 cells was significantly reduced by NDRG2 expression $(\mathrm{P}<0.01)$. In addition, $82.1 \%$ of NDRG2-expressing cells were in S-phase, compared to $74.4 \%$ in the control virus-infected cells $(\mathrm{P}<0.05)$. Furthermore, upregulation of NDRG2 induced an increase in oncosis, rather than apoptosis, in T24 cell. In conclusion, the results of the present study indicated that NDRG2 expression in mitochondria may arrest bladder cancer cells in S-phase as well as decrease cell proliferation through inducing oncosis. It was therefore proposed that NDRG2 was not only a biomarker, but also a tumor suppressor for bladder cancer.
\end{abstract}

\section{Introduction}

Urinary bladder cancer is ranked as the fifth most prevalent type of non-cutaneous neoplasm in the industrialized

Correspondence to: Dr Zhou Wu, Cancer Center, Affiliated Hospital of Guangdong Medical College, 57 South Renming Road, Zhanjiang, Guangdong 524001, P.R. China

E-mail: zhouaqwu@163.com

Key words: N-Myc downstream-regulated gene 2, proliferation, $\mathrm{T} 24$ cells, oncosis world, which accounts for $>4 \%$ of all cancer diagnoses worldwide (1). Urinary bladder cancer presents in two forms: Superficial tumors and muscle-invasive cancers. Localized, organ-sparing therapeutic techniques may be used for the treatment of superficial tumor (stages Ta and T1) tissue (2); however, muscle-invasive cancers (stage $\mathrm{T} 2+$ ) require immediate radical treatment. Superficial tumors have a $\leq 60 \%$ risk of progressing to muscle-invasive cancer, as reported in a 15 -year follow-up study (3); therefore, this group is the primary focus of clinical interest (4). Altered p53 gene expression and p53 pathways were reported to be present in muscle-invasive bladder cancer (T2-T4) and advanced stages (2); in addition, irregular p53 activity was demonstrated to be associated with augmented angiogenesis, invasiveness, metastasis and recurrence, resulting in poor prognoses (5). However, conflicting evidence has been reported on whether p53 mutations induce increased responsiveness or resistance to cisplatin-based systemic chemotherapy in bladder cancer (6).

N-Myc downstream-regulated gene 2 (NDRG2), a novel p53-inducible gene, was suggested to be involved in the DNA damage-induced p53-associated apoptotic pathway as well as to have a role in inhibiting proliferation (7). An increase in NDRG2 expression was reported to occur during cell differentiation, aging and the onset of diabetes. In addition, a positive correlation was observed between NDRG2 and tumor differentiation, as well as a negative correlation between NDRG2 and tumor invasion depth or Dukes' stage of adenocarcinoma $(8,9)$. Furthermore, reduced NDRG2 was reported to occur in numerous types of breast cancer, gastric cancer and low-grade glioblastomas. By contrast, NDRG2 overexpression was demonstrated to arrest HepG2 cells in M phase of the cell cycle. Several M phase-specific drugs, such as paclitaxel, are used in tumor chemotherapy. NDRG2 overexpression in HepG2 cells was therefore suggested to enhance the efficacy of these drugs (10).

To the best of our knowledge, the association between NDRG2 and bladder cancer cells remains to be investigated. The present study aimed to determine the effects of NDRG2 expression on T24 human bladder cancer cells. T24 cells have a p53 mutation with an in-frame deletion of tyrosine 126 and were derived from an undifferentiated grade III bladder carcinoma. This in vitro model was used to determine whether NDRG2 overexpression had an effect on the proliferation and cell cycle progression of T24 cells as well as to identify the subcellular localization of NDRG2 expression. In addition, the 
present study aimed to elucidate the function of NDRG2 as a tumor suppressor in bladder cancer.

\section{Materials and methods}

Cell culture. T24 human bladder cancer cells (Shanghai Institute of Cell Biology, Chinese Academy of Science, Shanghai, China) were cultured in RPMI 1640 medium (Life Technologies, Grand Island, NY, USA) supplemented with $10 \%$ heat-inactivated fetal bovine serum (Life Technologies), $100 \mathrm{U} / \mathrm{ml}$ penicillin and $100 \mathrm{mg} / \mathrm{l}$ streptomycin (Life Technologies) in a humidified atmosphere containing $5 \% \mathrm{CO}_{2}$ maintained at $37^{\circ} \mathrm{C}$.

Constructs and transfection. Recombinant adenoviruses, pAV.EX1d-NDRG2/internal ribosome entry site (IRES)/enhanced green fluorescent protein (eGFP) and control pAV.EX1d-eGFP, were constructed as previously described (11) using Gateway technology (Life Technologies). Briefly, NDRG2 was amplified by PCR using Phusion High-Fidelity DNA Polymerase (Thermo Fisher Scientific, Waltham, MA, USA) to construct a Gateway entry clone: pDown-NDRG2/IRES/eGFP (Cyagen Biosciences Inc., Guangzhou, China), which was followed by recombination with pAV/Des1d (Cyagen Biosciences Inc.) destination vector, in order to create the pAV.EX1d-NDRG2/IRES/eGFP adenoviral expression vector. The recombinant pAV.EX1d/eGFP without the NDRG2 insert was constructed and used as a control viral vector. For T24 cell infection, adenoviral particles containing 40 multiplicity of infection (MOI) were experimentally determined for T24 cells and used in the following experiments. MOI was determined using Adeno-X Rapid Titer kit (Clontech Laboratories, Inc., Mountain View, CA, USA), according to the manufacturer's instructions. T24 cells were seeded into six-well plates at a density of $5 \times 10^{5}$ cells/well and incubated at $37^{\circ} \mathrm{C}$ and $5 \%$ $\mathrm{CO}_{2}$ overnight until the cells had reached $\sim 80 \%$ confluence. NDRG2 (T24-NDRG2) or the control gene GFP (T24-GFP) were added to T24 cells with serum-free medium. Cells were incubated at $37^{\circ} \mathrm{C}$ and $5 \% \mathrm{CO}_{2}$ for $16 \mathrm{~h}$, the medium was then replaced with growth medium and incubated for different periods of time, as indicated. Non-infected control (T24) cells were also included in the present study.

Western blot analysis. The cells were lysed using $1 \mathrm{X}$ ice-cold radioimmunoprecipitation buffer (Abcam, Cambridge, MA, USA). Total proteins in the supernatant were collected after 20 min centrifugation at $16,000 \mathrm{x} \mathrm{g}$ at $4^{\circ} \mathrm{C}$, and the protein concentration was measured using a bicinchoninic acid protein assay (Pierce Biotechnology Inc., Rockford, IL, USA). A total of $100 \mu \mathrm{g}$ lysate was loaded per lane onto $10 \%$ SDS polyacrylamide gels (Bio-Rad Laboratories, Inc., Hercules, CA, USA) for separation by electrophoresis and then transferred onto nitrocellulose membranes (GE Healthcare, Piscataway, NJ, USA). Membranes were then incubated with $5 \%$ fat-free milk in Tris-buffered saline containing $0.05 \%$ Tween-20 (Sigma-Aldrich, St. Louis, MO, USA) for $1 \mathrm{~h}$ at room temperature. Polyclonal mouse anti-NDRG2 (Abnova, Taipei, Taiwan) was then added and incubated overnight at $4^{\circ} \mathrm{C}$. Following washing three times with phosphate-buffered saline (PBS), membranes were incubated with horseradish peroxidase-conjugated rabbit anti-mouse secondary antibody (1:1,000; Abcam, Cambridge, MA, USA) for $1 \mathrm{~h}$ at room temperature. Rabbit anti-human NADPH (1:1,000; Abcam) was used as a control. The blots were developed using SuperSignal West Pico Stable Peroxide Solution (Pierce Biotechnology, Inc.) and exposed to X-ray film (Fujifilm, Tokyo, Japan) using the Konica SRX-101 Processor (Konica Minolta Medical Imaging USA, Inc., Wayne, NJ, USA) for visualization.

Cell growth assays. Cell growth was monitored using an MTT assay. In brief, cells were seeded onto 96-well plates at an initial density of $2 \times 10^{3}$ cells/well (experiments were performed in triplicate). At each time point $(1,2,3,4,5,6$, and 7 days post-transfection), cells were washed and incubated with culture medium containing $0.5 \mathrm{mg} / \mathrm{ml}$ MTT (Sigma-Aldrich) at $37^{\circ} \mathrm{C}$ for $4 \mathrm{~h}$. The culture medium was removed by aspiration and $150 \mu$ l dimethyl sulfoxide was added to each well. The absorbance (optical density, OD) of the reaction solution was measured at $570 \mathrm{~nm}$ using a Synergy H4 Hybrid microplate reader (BioTek Instruments, Winooski, VT, USA).

Cell cycle analysis. Cells were seeded in 60-mm diameter plates $\left(6 \times 10^{5}\right.$ cells/plate) with complete medium and incubated at $37^{\circ} \mathrm{C}$ and $5 \% \mathrm{CO}_{2}$ overnight. The cells were then cultured in serum-free medium for $48 \mathrm{~h}$, in order to synchronize the cells, the medium was subsequently replaced with complete medium for a further $24 \mathrm{~h}$. Cells were then washed with ice-cold PBS and suspended in $\sim 0.5 \mathrm{ml} 70 \%$ alcohol prior to incubation at $4^{\circ} \mathrm{C}$ for $30 \mathrm{~min}$. The suspension was filtered through a $50 \mathrm{~mm}$ nylon mesh (BD Biosciences, San Jose, CA, USA), and the DNA content of propidium iodide (Life Technologies)-stained nuclei was analyzed using a flow cytometer (EPICS XL; Beckman Coulter, Inc., Miami, FL, USA). Cell cycle analysis was then performed using MultiCycle AV OCX DNA Cell Cycle Analyzed Software (Phoenix Flow Systems, San Diego, CA, USA). The proliferous index $(P I)$ was calculated as: $P I=\left(S+G_{2}\right) /\left(S+G_{2}+G_{1}\right)(12)$.

NDRG2 subcellular localization analysis. Cells $\left(5 \times 10^{4}\right)$ were grown on glass coverslips at $37^{\circ} \mathrm{C}$ and $5 \% \mathrm{CO}_{2}$ overnight. At different time points following transfection, cells were incubated for $30 \mathrm{~min}$ at $37^{\circ} \mathrm{C}$ with: Rhodamine 123 , a cell-permeable mitochondria-selective dye, (Invitrogen Life Technologies, Carlsbad, CA, USA); Bodipy (Life Technologies), a cell-permeable golgi apparatus-selective dye; or Lucifer Yellow (Life Technologies), a cell-permeable lysosome-selective dye. Cells were then rinsed with PBS three times and fixed in freshly prepared $4 \%$ paraformaldehyde (Santa Cruz Biotechnology, Inc., Dallas, TX, USA) in PBS for $30 \mathrm{~min}$ at $4^{\circ} \mathrm{C}$. The glass coverslips were then placed on the glass slides and covered with the cover glasses with mounting medium (Life Technologies). Slides were observed under a confocal laser fluorescence microscope (Zeiss 710; Carl Zeiss, Oberchoken, Germany).

Immunofluorescence assay (IFA). Parental T24 cells and lentivirus-infected T24 cells were cultured in Nunc Lab-Tek 4-well chamber slides (Thermo Fisher Scientific) overnight 
at $37^{\circ} \mathrm{C}$ with $5 \% \mathrm{CO}_{2}$. The cells were then fixed with $4 \%$ paraformaldehyde, washed with PBS, and permeabilized in $0.2 \%$ Tritron X-100 at room temperature for $5 \mathrm{~min}$. Post-permeabilization, mouse anti-NDRG2 (Abnova), diluted in PBS containing 1\% BSA (1:100 dilution), was added. After a $1 \mathrm{~h}$ incubation, the cells were washed with PBS, followed by incubation with Alexa Fluor 594-labeled rabbit anti-mouse secondary antibody (Life Technologies). The cells were subseqently washed with PBS, and mounted with anti-fade mounting medium containing DAPI (Life Technologies). Staining was examined using a Zeiss 710 fluorescence microscope.

Colony formation assay. Colony formation assay was performed, according to a previously described procedure (13). Briefly, 100 cells were plated in each well of 6-well culture plates, with triplicate repeats for each sample. The cells were grown at $37^{\circ} \mathrm{C}$ and $5 \% \mathrm{CO}_{2}$ for 14 days and then fixed and stained with $0.5 \%$ crystal violet. Colony counting was conducted under a Leica Mil microscope (Leica Microsystems Inc., Buffalo Grove, IL, USA).

Oncosis analysis. One day post-infection, the T24 cells infected with NDRG2 and control GFP viruses, and their parental cells, were cultured in 24-well plate for $24 \mathrm{~h}$. The cells were grown for $24 \mathrm{~h}$ and then stained with NucBlue Live Ready Probes kit (Life Technologies), according to the manufactuer's instructions. Cell morphology was visualized using a Olympus IX1 microscope (Olympus Corporation, Tokyo, Japan).

Statistical analysis. Values are expressed as the mean \pm standard deviation. SPSS 11.5 software (SPSS, Inc., Chicago, IL, USA) was used to perform one-way analysis of variance followed by Student t-tests for independent groups. The data were subjected to Bonferroni's correction when necessary. The $\chi^{2}$ or Fisher exact tests were used to assess dichotomous variables. $\mathrm{P}<0.05$ was considered to indicate a statistically significant difference between values.

\section{Results}

Upregulation of NDRG2 in T24 cells. In order to validate the success of infection, cell morphology was observed using a light microscope and fluorescence microscope. The results showed that $\sim 90 \%$ cells expressed GFP in the pAV.EX1d-eGFP-infected group; by contrast, only $\sim 60 \%$ cells expressed GFP in the pAV. EX1d-NDRG2/IRES/eGFP-infected group (Fig. 1A and B).

In addition, NDRG2 protein levels were determined using western blot and IFA analyses. In normal cultured T24 cells, low levels of NDRG2 expression were detected. However, following infection with the recombinant GFP-NDRG2 adenovirus, NDRG2 levels were significantly elevated, while low levels of NDRG2 were detected in cells infected with the GFP control virus (Fig. 2A and B).

Localization of NDRG2 in T24 cells. In order to evaluate the subcellular localization of NDRG2, cells were stained with organelle-specific dyes and then visualized using a confocal laser fluorescence microscope. As shown in Fig. 3A, following infection with the GFP-NDRG2 virus, NDRG2 was observed to be expressed primarily in the mitochondria and was not co-localized with the golgi apparatus or lysosomes. In addition, the expression of NDRG2 in the mitochondria was observed to be increased significantly from at 24,48 and $72 \mathrm{~h}$ post infection compared with $6 \mathrm{~h}$ post infection $(\mathrm{P}<0.05,0.05$ and 0.01 , respectively) (Fig. 3B).

NDRG2 inhibits T24 cell proliferation. Colony formation assays were used to demonstrate that NDRG2 expression significantly inhibited the colony formation of T24 cells following 2 weeks of culture (Fig. 4A), with colony formation efficiencies of 19.6, 63.1 and $74.5 \%$ for T24-NDRG, T24-GFP and T24 cells, respectively (Table I).

An MTT assay was then used to determine cell proliferation. Although cells from all experimental groups continually grew between days 1 and 7, the growth curve for cells infected with GFP-NDRG2 was significantly lower compared with those of the cells infected with control GFP and untransfected cells $(\mathrm{P}<0.05)$ (Fig. 4B). This suggested that NDRG2 had the potential to inhibit the proliferation of T24 cells.

NDRG2 induces the cell cycle arrest of T24 cells. In order to further investigate the mechanisms by which NDRG2 inhibits T24 cell growth, the effects of NDRG2 expression on cell cycle progression were investigated using fluorescence-activated cell sorting analysis. The results of the cell cycle analysis revealed that $82.1 \%$ of GFP-NDRG2-transfected cells were in S phase compared with $74.4 \%$ of control virus-infected cells, whereas $14.9 \%$ of GFP-NDRG2-transfected cells were in $\mathrm{G}_{1}$ phase compared with $20.5 \%$ of untransfected cells $(\mathrm{P}<0.05)$ (Fig. 5).

NDRG2 induces T24 cell oncosis. At $48 \mathrm{~h}$ post infection, an increased number of cells in the T24-NDRG2-transfected group demonstrated features of oncosis, including swelling and nuclear chromatin clumping, compared with the T24-GFP group (Fig. 6).

\section{Discussion}

p53, encoded by the human gene TP53, is a well-known tumor suppressor, the inactivation of which was reported to be associated with tumor progression, metastasis and an aggressive phenotype of bladder cancer (14). A previous study indicated that small interfering RNA knockdown of mutant p53 induced cell cycle arrest at $G_{2}$ phase as well as promoted apoptosis in T24 human bladder cancer cells (9). NDRG2, a novel p53-inducible gene, was suggested to be involved in the DNA damage-induced p53-associated apoptotic pathway (7). To the best of our knowledge, the present study was the first to identify the role of NDRG2 in bladder cancer.

Several studies have reported negative correlations between the expression of NDRG2 and tumor growth or metastasis in renal and breast cancer (12,15-17). Multivariate analysis revealed that loss of NDRG2 expression was a significant and independent prognostic indicator of gastric carcinoma (18). The current study demonstrated that NDRG2 expression was low in T24 bladder cancer cells, while upregulated NDRG2 expression inhibited cancer cell proliferation, indicating that bladder cancer proliferation may be attributed to NDRG2 deficiency. These results therefore indicated that, NDRG2 may be considered as a novel target for bladder cancer therapy. 
A

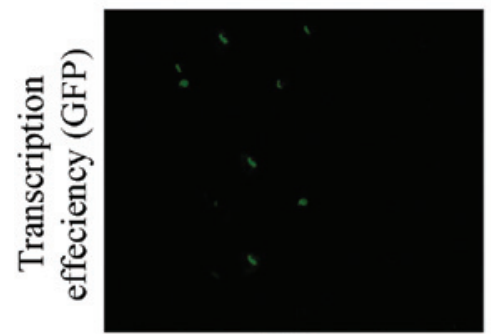

$\mathrm{T} 24$

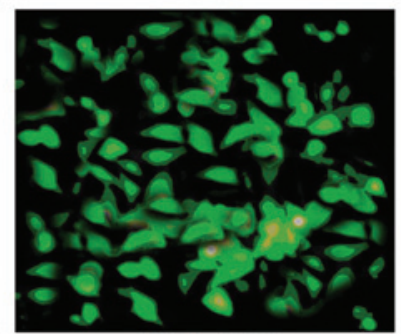

T24-GFP

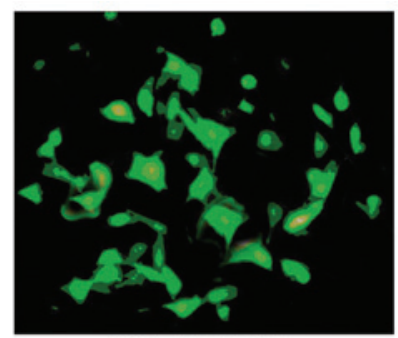

T24-NDRG2

B

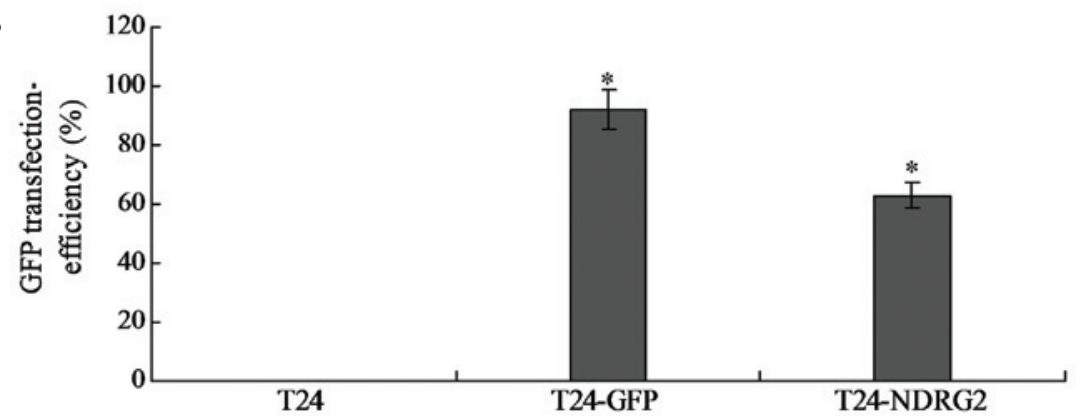

Figure 1. Validation of transfection efficiency in T24 cells using fluorescence microscopy. (A) Representative images of GFP expression in T24, T24-GFP and T24-NDRG2 cells. (B) Quantification of GFP gene transfection efficiency. Values are presented as the mean \pm standard deviation. ${ }^{*} \mathrm{P}<0.01$ vs. T24 cells. GFP, green fluorescent protein; T24-GFP, control GFP adenovirus-infected T24 cells; T24-NDRG2, GFP-N-Myc downstream-regulated gene 2 adenovirus-infected T24 cells.

A

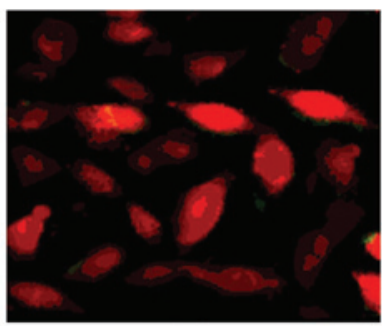

T24

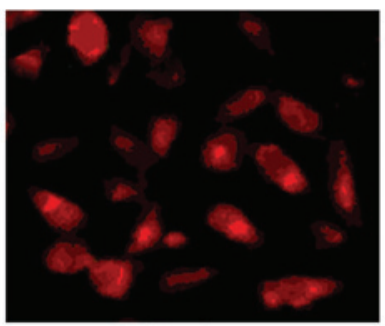

T24-GFP

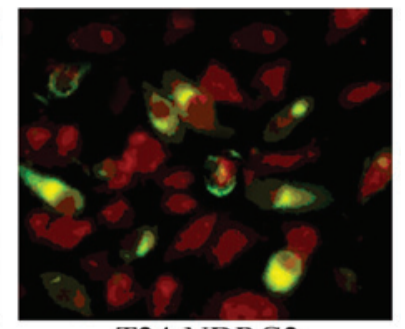

T24-NDRG2

B
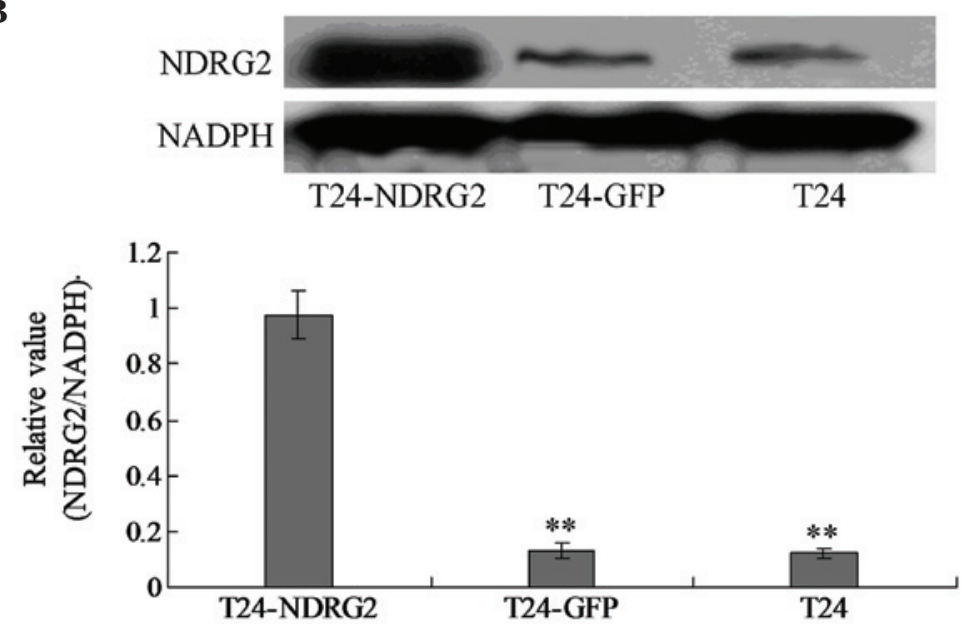

Figure 2. Examination of NDRG2 protein expression in T24 cells using western blot analysis and IFA detection. (A) IFA assay for the NDRG2 protein expression in T24, T24-GFP and T24-NDRG2 cells. (B) Western blot analysis and quantification for NDRG2 protein expression in T24, T24-GFP and T24-NDRG2cells. NADPH was used as a loading control. Values are presented as the mean \pm standard deviation. ${ }^{* *} \mathrm{P}<0.01$ vs. T24 cells. NDRG2, N-Myc dowstream-regulated gene 2; T24-GFP, control green fluorescent protein adenovirus-infected T24 cells; T24-NDRG2, GFP-NDRG2 adenovirus-infected T24 cells.

NDRG2 protein exists primarily in the cytoplasm; however, it has also been associated with the cell membrane and adherent junctions (19). One study demonstrated that following DNA damage, NDRG2 translocated from the cytoplasm to the nucleus (20). Wang et al reported that a specific section of NDRG2 (amino acids 101 to 178) was responsible 
Table I. Cloning efficiency of untransfected T24 cells and T24 cells transfected with GFP or GFP-NDRG2 adenoviruses.

\begin{tabular}{lcc}
\hline Cell type & $\begin{array}{c}\text { Number of } \\
\text { cloning cells (n) }\end{array}$ & $\begin{array}{c}\text { Cloning efficiency } \\
\text { (numbers/500 cells) }\end{array}$ \\
\hline T24 & 382 & 0.745 \\
T24-GFP & 258 & 0.631 \\
T24-NDRG2 & 124 & 0.196 \\
\hline
\end{tabular}

Cloning efficiency was determined day 14 post-transfection. GFP, green fluorescent protein; NDRG2, N-Myc downstream-regulated gene 2; T24-GFP, control GFP adenovirus-infected T24 cells; T24-NDRG2, GFP-NDRG2 adenovirus-infected T24 cells.

A

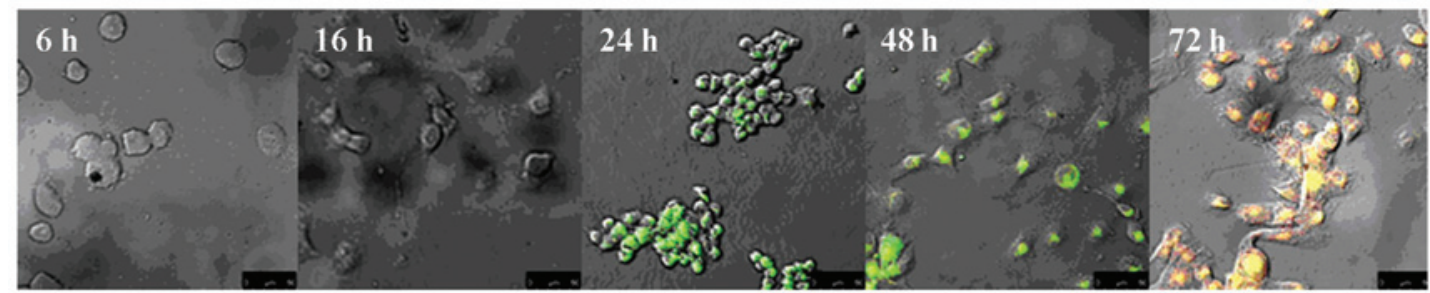

$\mathbf{B}$

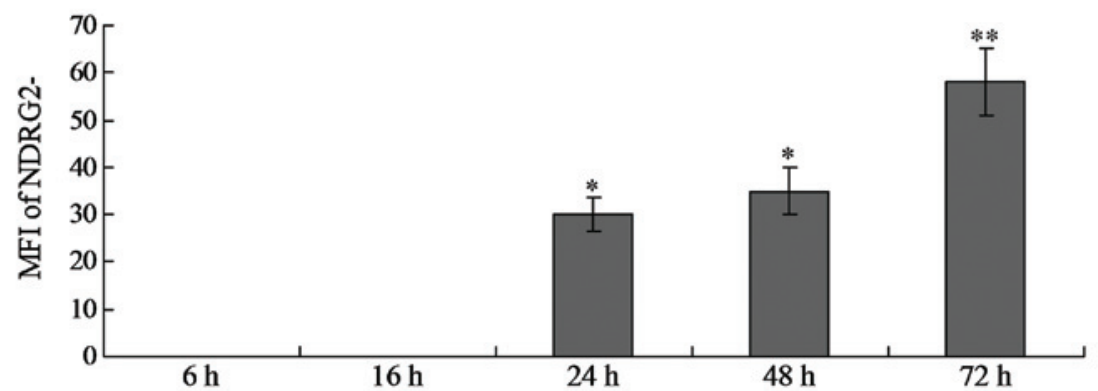

Figure 3. Subcellular localization of NDRG2 protein following gene transfection. (A) Subcellular localization of NDRG2 protein in the mitochondria of the NDRG2-transfected cells at 6,16,24, 48 and $72 \mathrm{~h}$ following transfection. (B) Quantification of NDRG2 protein expression following transfection. Values are presented as the mean \pm standard deviation. ${ }^{*} \mathrm{P}<0.05$ and ${ }^{* *} \mathrm{P}<0.01$ vs. $6 \mathrm{~h}$. NDRG2, N-Myc dowstream-regulated gene 2; MFI, mean fluorescence intensity.

A

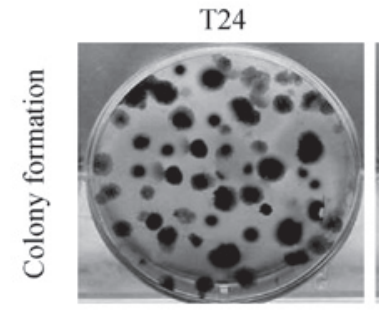

T24-GFP

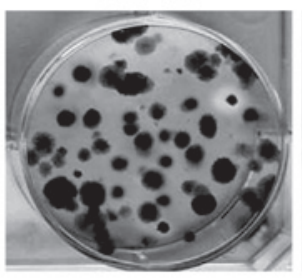

T24-NDRG2

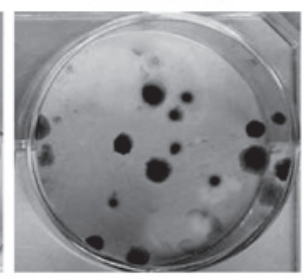

B

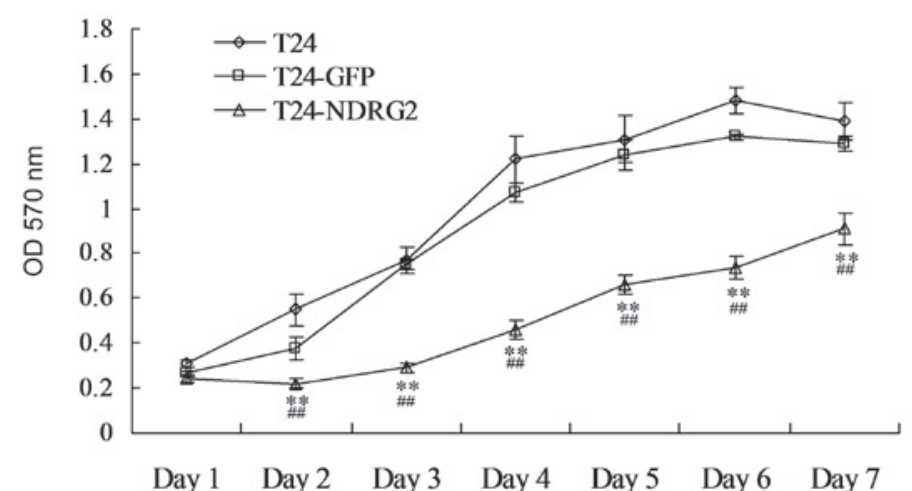

Figure 4. Overexpression of NDRG2 inhibits the growth of T24 cells. (A) Colony formation assay for proliferation in T24, T24-GFP and T24-NDRG2 cells following 2 weeks of culture. (B) An MTT assay was performed in order to quantify cell proliferation at day 1, 2, 3, 4, 5, 6 and 7 following transfection. Values are presented as the mean \pm standard deviation. ${ }^{* * *} \mathrm{P}<0.01$ vs. T24; ${ }^{\# \#} \mathrm{P}<0.01$ vs. T24-GFP. NDRG2, N-Myc dowstream-regulated gene 2; T24-GFP, control green fluorescent protein adenovirus-infected T24 cells; T24-NDRG2, GFP-NDRG2 adenovirus-infected T24 cells. 
A

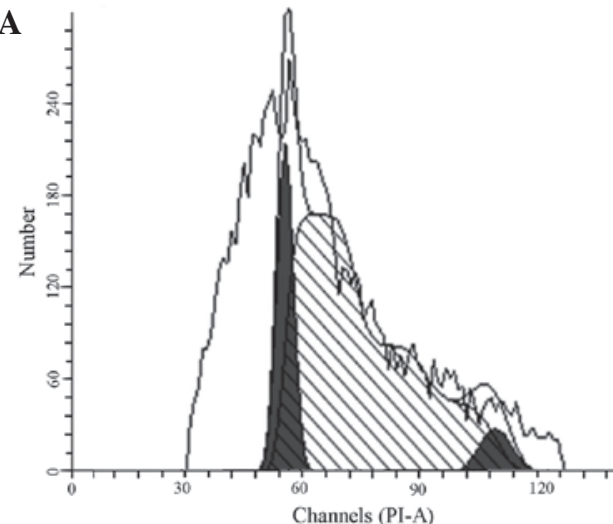

C

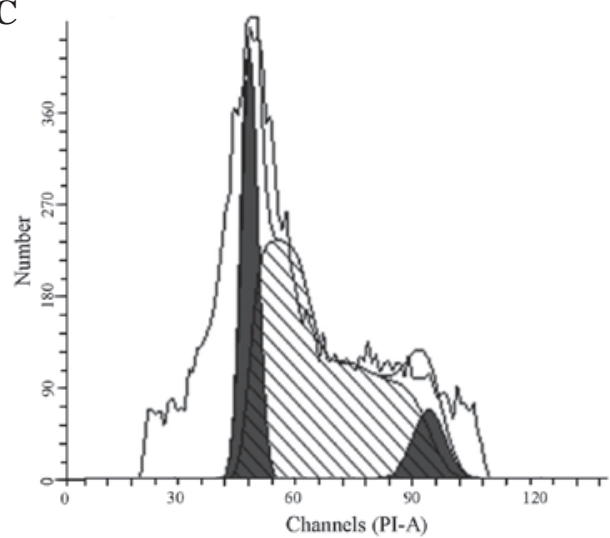

B

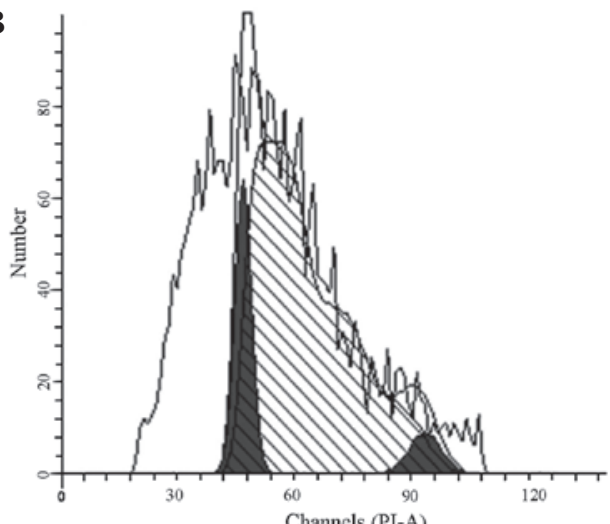

D

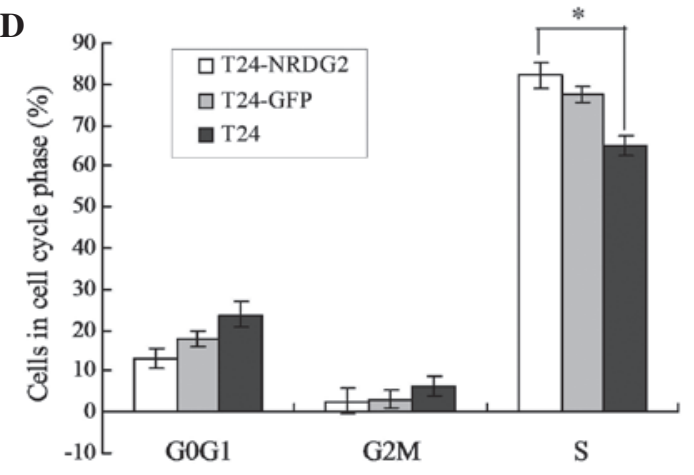

Figure 5. NDRG2 induces cell cycle arrest in T24 cells. Flow cytometry was used for cell cycle analysis in (A) T24-NDRG2, (B) T24-GFP and (C) T24 cells. (D) Quantification of the proportion of cells in $\mathrm{G}_{0} / \mathrm{G}_{1}, \mathrm{G}_{2} \mathrm{M}$ and $\mathrm{S}$ phase of the cell cycle. Values are presented as the mean \pm standard deviation. " $\mathrm{P}<0.01 \mathrm{vs}$. T24 cells. NDRG2, N-Myc dowstream-regulated gene 2; T24-GFP, control green fluorescent protein adenovirus-infected T24 cells; T24-NDRG2, GFP-NDRG2 adenovirus-infected T24 cells; PI-A, propidium iodide-A.

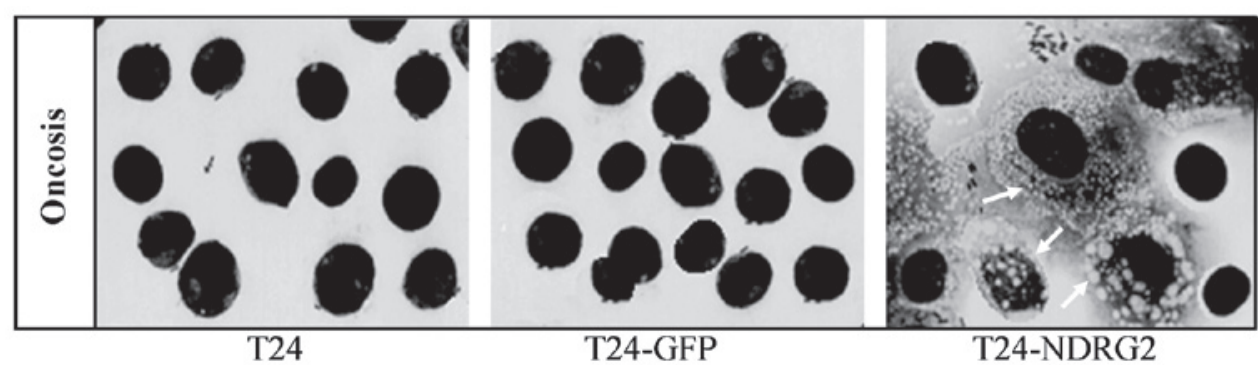

Figure 6. NDRG2 transfection triggers oncosis in T24 cells. Oncosis was not observed in T24 and T24-GFP cells, whereas in the T24-NDRG2 group, cells undergoing oncosis were detected. Arrows indicate the oncotic cells. NDRG2, N-Myc dowstream-regulated gene 2; T24-GFP, control green fluorescent protein adenovirus-infected T24 cells; T24-NDRG2, GFP-NDRG2 adenovirus-infected T24 cells.

for its nuclear translocation (21). It was suggested that nuclear NDRG2 expression may affect cancer biology. However, in current study, NDRG2 was primarily expressed in the mitochondria, the levels of which increased in a time-dependent manner. It has been reported that mitochondrial function is impaired in cancer cells (22). The metabolism of proliferative cancer cells alters due to active metabolic reprogramming induced by oncogenes and tumor suppressors, which is predominantly dependent on mitochondria, as they are functional biosynthetic organelles (23). In proliferating cells, the production of adenosine triphosphate through oxidative phosphorylation is secondary to the use of mitochondrial enzymes in the synthesis of anabolic precursors $(23,24)$. Phosphoinositide 3-kinase (PI3K)/Akt/mammalian target of rapamycin complex 1 pathway activation drives anabolic metabolism and tumor genesis through mitochondria reprogramming (23). Overexpression of NDRG2 in malignant breast cancer cells was reported to inhibit Akt phosphorylation as well as induce p38 mitogen-activated protein kinase and stress-activated protein kinase/c-Jun N-terminal kinase phosphorylation (25). Meanwhile, Janus kinase 2 or signal transducer and activator of transcription 3 activation in resting and insulin-like growth factor-stimulated cells was inhibited by NDRG2 expression; this therefore indicated that NDRG2 may act as a growth inhibitory gene in the signal transduction pathways of breast tumor cells (26). However, further studies are required in order to elucidate whether NDRG2 regulates mitochondria reprogramming via the PI3K/Akt pathway. 
The present study demonstrated that the tumor suppressor activity of NDRG2 was regulated through cell cycle progression arrest in S phase. Kim et al demonstrated that NDRG2 was able to attenuate cell proliferation via the suppression of activator protein-1 activity in human colon carcinoma cells (27). In addition, it was reported that NDRG2 controlled the cell cycle through regulating intracellular signals via the phosphorylation pathway of cyclin D1 expression (27); this may provide an explanation for the altered cell cycle activity observed in the present study. Another previous study revealed that NDRG2 promoted the accumulation of cancer cells in $\mathrm{G}_{1}$ phase and in turn decreased the number of cells in $\mathrm{S}$ phase of the cell cycle in A-498 renal cancer cells, which corresponded with a reduction in cyclin D1 and cyclin E expression (12). Furthermore, the present study demonstrated that NDRG2 induced bladder cancer cell apoptosis by increasing the accumulation of $\mathrm{T} 24$ cells in S phase.

In order to explore the mechanism of NDRG2-inhibited T24 cell proliferation, cell death was monitored in the T24 cells. Former studies indicated that NDRG2 was unable to trigger apoptosis, autophagy, paraptosis and other cell-programmed death (28). Therefore, the present study investigated whether NDRG2 triggered oncosis, which is a specific cell death pathway. The result indicated the NDRG2 induced oncosis in T24 cells, which triggered the inhibition of proliferation.

In conclusion, the results of the present study indicated that NDRG2 was able to influence mitochondria function, arrest cells in $\mathrm{S}$ phase and decrease bladder cancer cell proliferation by inducing oncosis. These findings further enhance the current understanding of NDRG2 and its role in tumor cells and therefore may have important implications for NDRG2-targeted novel cancer therapies.

\section{Acknowledgements}

The present study was supported by the Project of Science and Technology Plan of Guangdong Province (no. 10B06090008063041; Guangdong, China).

\section{References}

1. Jemal A, Bray F, Center MM, et al: Global cancer statistics. CA Cancer J Clin 61: 69-90, 2011.

2. Kaufman DS, Shipley WU and Feldman AS: Bladder cancer. Lancet 374: 239-249, 2009.

3. Cookson MS, Herr HW, Zhang ZF, et al: The treated natural history of high risk superficial bladder cancer: 15-year outcome. J Urol 158: 62-67, 1997 .

4. Cheng L, Cheville JC, Neumann RM, et al: Survival of patients with carcinoma in situ of the urinary bladder. Cancer 85: 2469-2474, 1999.

5. Mitra AP, Datar RH and Cote RJ: Molecular pathways in invasive bladder cancer: new insights into mechanisms, progression and target identification. J Clin Oncol 24: 5552-5564, 2006.

6. Nishiyama H, Watanabe J and Ogawa O: p53 and chemosensitivity in bladder cancer. Int J Clin Oncol 13: 282-286, 2008.
7. Liu N, Wang L, Li X, et al: N-Myc downstream-regulated gene 2 is involved in p53-mediated apoptosis. Nucleic Acids Res 36: 5335-5349, 2008.

8. Lorentzen A, Vogel LK, Lewinsky RH, et al: Expression of NDRG2 is down-regulated in high-risk adenomas and colorectal carcinoma. BMC Cancer 7: 192, 2007.

9. Li R, Yu C, Jiang F, et al: Overexpression of N-Myc downstream-regulated gene 2 (NDRG2) regulates the proliferation and invasion of bladder cancer cells in vitro and in vivo. PLoS One 8: e76689, 2013.

10. Liu X, Niu T, Hou W, et al: Microarray profiling of HepG2 cells ectopically expressing NDRG2. Gene 503: 48-55, 2012.

11. Zheng J, Li Y, Yang J, et al: NDRG2 inhibits hepatocellular carcinoma adhesion, migration and invasion by regulating CD24 expression. BMC Cancer 251: 1-9, 2011.

12. Ma JJ, Liao CG, Jiang X, et al: NDRG2 suppresses the proliferation of clear cell renal cell carcinoma cell A-498. J Exp Clin Cancer Res 29: 103, 2010.

13. Franken NA, Rodermond HM, Stap J, Haveman J and van Bree C: Clonogenic assay of cells in vitro. Nat Protoc 1: 2315-2319, 2006.

14. Choi W, Czerniak B, Ochoa A, Su X, Siefker-Radtke A, Dinney $\mathrm{C}$ and McConkey DJ: Intrinsic basal and luminal subtypes of muscle-invasive bladder cancer. Nat Rev Urol 11: 400-410, 2014.

15. Hummerich L, Müller R, Hess J, et al: Identification of novel tumour-associated genes differentially expressed in the process of squamous cell cancer development. Oncogene 25: 111-121, 2006.

16. Liu N, Wang L, Liu X, et al: Promoter methylation, mutation and genomic deletion are involved in the decreased NDRG2 expression levels in several cancer cell lines. Biochem Biophys Res Co 358: 164-169, 2007.

17. Lee DC, Kang YK, Kim WH, et al: Functional and clinical evidence for NDRG2 as a candidate suppressor of liver cancer metastasis. Cancer Res 68: 4210-4220, 2008.

18. Choi SC, Yoon SR, Park YP, et al: Expression of NDRG2 is related to tumor progression and survival of gastric cancer patients through Fas-mediated cell death. Exp Mol Med 39: 705-714, 2007.

19. Boulkroun S, Fay M, Zennaro MC, et al: Characterization of rat NDRG2 (N-Myc downstream regulated gene 2), a novel early mineralocorticoid-specific induced gene. J Biol Chem 277: 31506-31515, 2002.

20. Zhang J, Liu J, Li X, et al: The physical and functional interaction of NDRG2 with MSP58 in cells. Biochem Biophys Res Commun 352: 6-11, 2007.

21. Wang L, Liu N, Yao L, et al: NDRG2 is a new HIF-1 target gene necessary for hypoxia-induced apoptosis in A549 cells. Cell Physiol Biochem 21: 239-250, 2008.

22. Cairns RA, Harris IS and Mark TW: Regulation of cancer cell metabolism. Nat Rev Cancer 11: 85-95, 2011.

23. Ward PS and Thompson CB: Metabolic reprogramming: a cancer hallmark even warburg did not anticipate. Cancer Cell 21: 297-308, 2012.

24. Dang CV. Links between metabolism and cancer. Genes Dev 26: 877-890, 2012.

25. Park Y, Shon SK, Kim A, et al: SOCS1 induced by NDRG2 expression negatively regulates STAT3 activation in breast cancer cells. Biochem Biophys Res Co 363: 361-367, 2007.

26. Yao L, Zhang J and Liu X: NDRG2: a Myc-repressed gene involved in cancer and cell stress. Acta Biochim Biophys Sin (Shanghai) 40: 625-635, 2008.

27. Kim YJ, Yoon SY, Kim JT, et al: NDRG2 suppresses cell proliferation through down-regulation of AP-1 activity in human colon carcinoma cells. Int J Cancer 124: 7-15, 2009.

28. Li T, Hu J, He GH, et al: Up-regulation of NDRG2 through nuclear factor-kappa B is required for leydig cell apoptosis in both human and murine infertile testes. Biochim Biophys Acta 1822: 301-313, 2012. 